\title{
FIRST OVERVIEW OF ORTHOPTERA AND MANTODEA OF THE SNIJEŽNICA KONAVOSKA MOUNTAIN
}

\author{
Fran Rebrina ${ }^{1,2}$ \& Nikola Tvrtković ${ }^{3}$ \\ ${ }^{1}$ Bijenička cesta 182, HR-10000 Zagreb, Croatia (e-mail: rebrinafran@gmail.com) \\ ${ }^{2} \mathrm{IUCN} / \mathrm{SSC}$ Grasshopper Specialist Group \\ ${ }^{3}$ Društvo za zaštitu prirode Hrvatske - NATURA, private address: Alagovićeva 21, \\ HR-10000 Zagreb, Croatia
}

\begin{abstract}
Rebrina, F, \& Tvrtković, N.: First overview of Orthoptera and Mantodea of the Sniježnica Konavoska Mountain, Nat. Croat. Vol. 28, No 1, 131-146, 2019, Zagreb.

During a study conducted in 2018 on Mt Sniježnica,the faunistically understudied southernmost karst mountain in Croatia, altogether 42 orthopteran and three mantid species were recorded at 18 localities at different altitudes. Two Poecilimon taxa new to Croatian fauna were found, one of them an undescribed species from the elegans group. Three bush-cricket species, namely Leptophyes intermedia, Platycleis albopunctata grisea and Pholidoptera fallax, were recorded for the first time in the Dubrovnik region. A curious variability of male cerci length, shape and position was observed within the Pachytrachis gracilis populations in the study area. The presence of several orthopteran species of importance for nature conservation was confirmed on Mt Sniježnica.
\end{abstract}

Key words: bush-crickets, Poecilimon, Pachytrachis, grasshoppers, mantids, Eastern Adriatic coast

Rebrina, F, \& Tvrtković, N.: Prvi pregled Orthoptera i Mantodea planine Sniježnice Konavoske, Nat. Croat. Vol. 28, No. 1, 131-146, 2019, Zagreb.

Tijekom istraživanja provedenog 2018. godine na Sniježnici, faunistički slabo istraženoj najjužnijoj krškoj planini u Hrvatskoj, na 18 lokaliteta na različitim nadmorskim visinama zabilježene su ukupno 42 vrste ravnokrilaca i tri vrste bogomoljki. Pronađene su dvije svojte roda Poecilimon nove za Hrvatsku, a jedna je od njih još neopisana vrsta iz elegans grupe. Tri vrste konjica, Leptophyes intermedia, Platycleis albopunctata grisea i Pholidoptera fallax, zabilježene su po prvi put na području Dubrovnika. U populacijama vrste Pachytrachis gracilis u istraživanom području primijećena je neobična varijabilnost dužine, oblika i položaja začanih nastavaka mužjaka. Potvrđena je prisutnost nekoliko vrsta ravnokrilaca od važnosti za propisivanje mjera očuvanja prirode na planini Sniježnici.

Ključne riječi: konjici, Poecilimon, Pachytrachis, skakavci, bogomoljke, istočna jadranska obala

\section{INTRODUCTION}

Sniježnica Konavoska Mountain, with the highest peak Sv. Ilija [St Elias], 1234 $\mathrm{m}$ a.s.l., is the southernmost Croatian karst mountain. The first faunistic research of the terrestrial fauna on the mountain started relatively late, in September 1984 (Croatian Natural History Museum, Branko Jalžić and N. Tvrtković) with the finding of a relict vole Dinaromys bogdanovi and an endangered snake Elaphe quatuorlineata. Italian orthopterist Filippo Maria Buzzetti was the first to note 20 grasshopper and bush-cricket species from Kuna Konavoska village and along the trail to Sv. Ilija in 2005 (Buzzettr, 2006); however, he published only the data for Arcyptera brevipennis brevipennis, Prionotropis hystrix hystrix, Barbitistes yersini, Saga pedo and Pachytrachis bosniacus. After a review of important faunistic data (TvrtKović \& Veen, 2006), Mt Sniježnica and Konavosko polje (karst field) 
were included in the Croatian Ecological Network in 2007 by the State Institute of Nature Protection (www.min.kulture/priroda/prilog1.2.novo/pdf), and were recently marked under the number HR2000964 (www.bioportal.hr). The snakes Elaphe quatuorlineata and Zamenis situla, and the relict vole Dinaromys bogdanovi, are the only terrestrial species from Mt Sniježnica included in the list of species important for protection. When it comes to orthopterans, KARAMAN et al. (2011) identified Troglophilus cavicola collected in 1995 by Marijana Franičević in Špilja Jezero cave and in 2006 by Roman Ozimec in Glogova jama pit (both situated on the slopes of Mt Sniježnica), as well as T. ovuliformis collected by Roman Ozimec in Špilja Jezero cave in 2002. After an examination of the Buzzetti Collection in Arzignano, Italy, J. Skejo and F. Rebrina redetermined a Pachytrachis specimen (previously identified as P. bosniacus) from Kuna Konavoska as P. gracilis (SкеJo et al., 2018), a continental species never found this close to the Adriatic coast before.

The present orthopterological survey in the vicinity of Dubrovnik, undertaken by the authors in 2018 with a particular focus on Sniježnica Konavoska Mt., was encouraged by several factors. Firstly, the faunistic knowledge of orthopteran insects in the south-easternmost part of Croatia is generally insufficient. More specifically, an old note existed about the finding of a specimen belonging to Poecilimon elegans Brunner von Wattenwyl, 1878 group in the Lovćen pass in Montenegro, with more than 70 smaller teeth in the apical part of the stridulatory file, differing from typical P. elegans specimens from Slovenia (Heller, 1988). Later, Ingrisch \& Pavićević (2010) discussed the existence of a probably new taxon with more than 100 stridulatory teeth from Montenegro (Lovćen pass) and from Mt Orjen, SE Grab, 1000 m a.s.l., in Bosnia and Hercegovina. SKEJo et al. (2018) presumed the presence of P. albolineatus from Montenegro and Bosnia and Hercegovina or another undescribed species (close to P. albolineatus) in the Dubrovnik region.

Accordingly, the main aims of the current study were: 1) to gain knowledge of the Orthoptera and Mantodea inhabiting Mt Sniježnica, both by reviewing the published data and performing a systematic orthopterological survey of the mountain, focusing on rare and/or previously unrecorded species; 2 ) to conduct preliminary research into the vertical distribution of Orthoptera and Mantodea on Mt Sniježnica.

\section{MATERIAL AND METHODS}

\section{Study area}

Mt Sniježnica is situated north of Konavosko polje, between the pass to Stravča/ Duba Konavoska and the Jablan Dol pass between Dubravka and Grab in Bosnia and Herzegovina. It is very close to the border with Montenegro, which is situated to the east (Fig. 1). The Zubci fault (geological fault, MARкоvić, 1971) stretches linearly in the N - S direction (Grab - Jablan Dol pass - Dubravka - Molunat peninsula), separating Mt Sniježnica Konavoska from the western slopes of Bjelotina ridge, the westernmost part of the large Orjen Mountain (1894 m a.s.l.) massif (https:// en.wikipedia.org/wiki/Orjen). The entire region has a Mediterranean climate but the Mt Orjen massif has abundant precipitation, with an annual average of 
about 50001 per $\mathrm{m}^{2}$. In the winter, the higher part of Sniježnica is often covered with snow (= snijeg in Croatian). The southern coast-oriented slopes and the central part of Mt Sniježnica Konavoska are located in Croatia, whereas the lower part of the northern slopes, in the direction of Popovo polje, is in Bosnia and Herzegovina. According to Marković (1971), the bedrock of the lower part of the southern slopes consists of Palaeogene formations, mostly flysch, sandstones and breccia. Above the flysch there is a layer of Triassic dolomite, while the bedrock in the higher portions of the southern slopes, from Kuna Konavoska to Sv. Ilija peak, is of Jurassic limestones. It forms a geomorphologically heterogeneous high karst plateau with sinkholes, hollows, pits, caves and several isolated peaks. The outback of Sv. Ilija peak area consists of Cretaceous limestones.

VuKELIĆ (2012) designated all forests in the south-eastern part of Croatia as Mediterranean deciduous forests and thickets with pubescent oak (Ordo Quercetalia pubescentis Klika 1933). In lower, warmer altitudes below $700 \mathrm{~m}$ a.s.l., sub-Mediterranean forests of the pubescent oak and Oriental hornbeam (Querco pubescentis - Carpinetum orientalis Horvatić 1939) grow on Mt Sniježnica, but on the higher, colder slopes above $700(-1000) \mathrm{m}$ a.s.l. and throughout the northern slopes, sub-Mediterranean montane forests of pubescent oak and hop hornbeam with yellow birthwort (Aristolochio luteae - Quercerum pubescentis (Horvat 1959) Poldini 2008) are found. Grasslands on deeper soils are rare, occurring only in

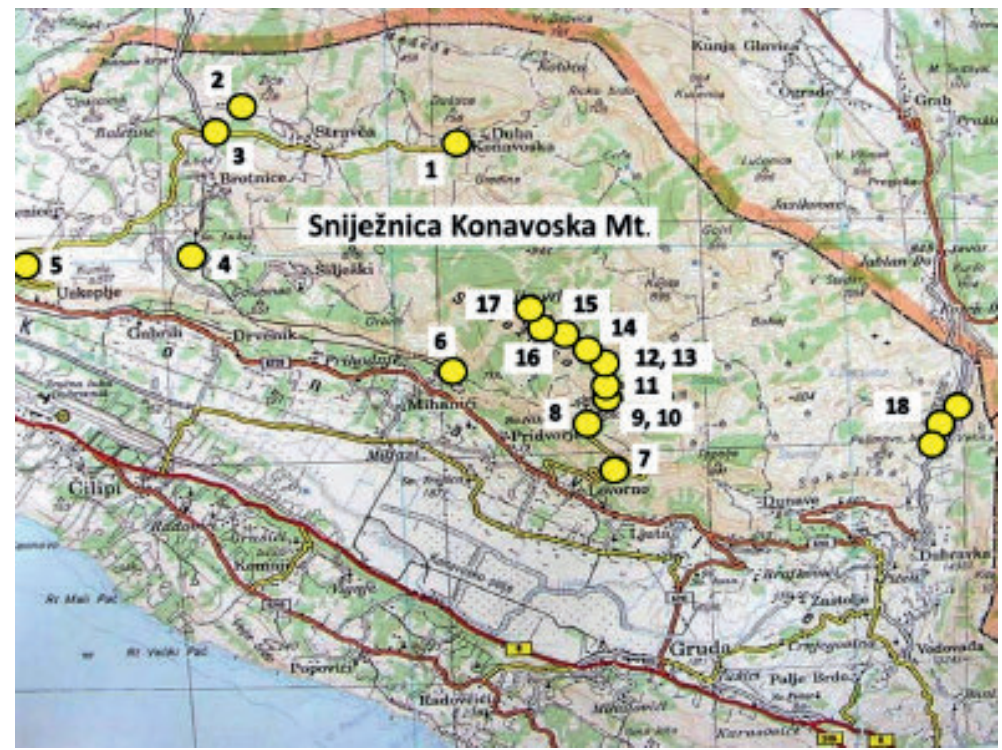

Fig. 1. Examined localities on Mt Sniježnica Konavoska, south of Dubrovnik City (Croatia): (1) Duba Konavoska, (2) Njivice, (3) fork Stravča - Njivice, (4) Sv. Luka, (5) Pass Konavle - Duba Konavoska, (6) Mihanići, tunel, (7) below pass to Kuna Konavoska, (8) Kuna Konavoska, before village, (9) Kuna Konavoska, start point to mountain trail, (10) trail Kuna Konavoska - Kupljenik, (11) Kupljenik, (12) trail Kupljenik - Ljeskovac, (13) Ljeskovac, (14) trail Ljeskovac - fork to Glogovac, (15) Glogovac, (16) trail fork to Glogovac - Sv. Ilija, (17) Sv. Ilija peak, (18) Prapratno transect. 
small karst depressions (sinkholes and hollows) like Kupljenik, Ljeskovac and Glogovac (Fig 1). However, on steep southern slopes, e.g. between Ljeskovac and Sv. Ilija peak, dry rocky pastures with bushes are common, today mostly in different successional stages due to the decline in the number of cattle, goats and sheep in villages (information obtained from local villagers). Around Sv. Ilija peak, there is a relatively small area of epi-Mediterranean dry rocky grassland.

\section{Sampling localities}

The study area was visited by the authors on two occasions in 2018, in July (21., 23.07.) and August (18., 20., 21.08.), during which most of the localities were investigated (Tab. 1). Caves and an abandoned tunnel were visited early in 2018 (16.05.) and again in 2019 (14.4.) by the second author and biospeleologist Branko Jalžić; however these visits were focused primarily on underground sites, which were investigated for cave crickets. In total, 18 localities at different altitudes on Mt Sniježnica were visited (Fig. 1, Tab. 1).

Tab. 1. Localities investigated during the study of Orthoptera and Mantodea of Mt Sniježnica (Croatia) in 2018 and 2019, with corresponding altitudes, geographic coordinates and visiting dates.

\begin{tabular}{|c|c|c|c|c|c|}
\hline No. & Locality & $\begin{array}{l}\text { Altitude } \\
\text { (m a.s.l.) }\end{array}$ & $\mathrm{N}$ & $\mathrm{E}$ & Date \\
\hline 1 & Duba Konavoska & 458 & $42^{\circ} 35^{\prime} 56,00^{\prime \prime}$ & $18^{\circ} 19^{\prime} 6,79^{\prime \prime}$ & 18.08.2018 \\
\hline 2 & Njivice: Jametina u dolinicah & 470 & $42^{\circ} 36^{\prime} 22,67^{\prime \prime}$ & $18^{\circ} 18^{\prime} 3,68^{\prime \prime}$ & 14.04.2019 \\
\hline 3 & Fork Stravča - Njivice & 490 & $42^{\circ} 36^{\prime} 10,72^{\prime \prime}$ & $18^{\circ} 17^{\prime} 48,77^{\prime}$ & 18.08.2018 \\
\hline 4 & Sv. Luka & 450 & $42^{\circ} 35^{\prime} 0,52^{\prime \prime}$ & $18^{\circ} 17^{\prime} 28,16^{\prime \prime}$ & 21.08.2018 \\
\hline 5 & Pass to Duba Konavoska & 500 & $42^{\circ} 35^{\prime} 2,97^{\prime \prime}$ & $18^{\circ} 15^{\prime} 31,40^{\prime \prime}$ & 18.08.2018 \\
\hline 6 & Mihanići (near the tunnel) & 330 & $42^{\circ} 33^{\prime} 43,04^{\prime \prime}$ & $18^{\circ} 20^{\prime} 25,1^{\prime \prime}$ & 16.05.2018 \\
\hline 7 & Pridvorje - Kuna Konavoska & 570 & $42^{\circ} 33^{\prime} 53,66^{\prime \prime}$ & $18^{\circ} 22^{\prime} 10,83^{\prime \prime}$ & 28.06 .2006 \\
\hline 8 & Kuna Konavoska, below the village & 670 & $42^{\circ} 33^{\prime} 21,64^{\prime \prime}$ & $18^{\circ} 21^{\prime} 48,61^{\prime \prime}$ & $\begin{array}{l}21.07 .2018 \\
21.08 .2018\end{array}$ \\
\hline 9 & $\begin{array}{l}\text { Kuna Konavoska, starting point of the trail to } \\
\text { Sv. Ilija peak }\end{array}$ & 700 & $42^{\circ} 33^{\prime} 35,61^{\prime \prime}$ & $18^{\circ} 21^{\prime} 43,19^{\prime \prime}$ & 21.07.2018 \\
\hline 10 & Trail Kuna Konavoska - Kupljenik & $710-800$ & & & $\begin{array}{l}21.07 .2018 \\
20.08 .2018\end{array}$ \\
\hline 11 & Kupljenik & 800 & $42^{\circ} 33^{\prime} 48,81^{\prime \prime}$ & $18^{\circ} 21^{\prime} 33,21^{\prime \prime}$ & 21.07.2018 \\
\hline 12 & Trail Kupljenik - Ljeskovac & $800-930$ & & & $\begin{array}{l}21.07 .2018 \\
20.08 .2018\end{array}$ \\
\hline 13 & Ljeskovac & 940 & $42^{\circ} 34^{\prime} 0,96^{\prime \prime}$ & $18^{\circ} 21^{\prime} 39,53^{\prime \prime}$ & $\begin{array}{l}21.07 .2018 \\
20.08 .2018\end{array}$ \\
\hline 14 & Trail Ljeskovac - fork to Glogovac & $940-1040$ & & & $\begin{array}{l}21.07 .2018 \\
20.08 .2018\end{array}$ \\
\hline 15 & Glogovac & $990-1000$ & $42^{\circ} 34^{\prime} 5,04^{\prime \prime}$ & $18^{\circ} 21^{\prime} 41,30^{\prime \prime}$ & 20.08.2018 \\
\hline 16 & Trail fork to Glogovac - Sv. Ilija peak & $1040-1180$ & & & $\begin{array}{l}21.07 .2018 \\
20.08 .2018\end{array}$ \\
\hline 17 & Sv. Ilija peak & 1230 & $42^{\circ} 34^{\prime} 26,39^{\prime \prime}$ & $18^{\circ} 21^{\prime} 6,26^{\prime \prime}$ & $\begin{array}{l}21.07 .2018 \\
20.08 .2018\end{array}$ \\
\hline 18 & $\begin{array}{l}\text { Prapratno valley transect (start and end point } \\
\text { noted) }\end{array}$ & 550 & $\begin{array}{l}42^{\circ} 32^{\prime} 55,5^{\prime \prime} \\
42^{\circ} 33^{\prime} 29,08^{\prime \prime}\end{array}$ & $\begin{array}{l}18^{\circ} 25^{\prime} 36,32^{\prime} \\
18^{\circ} 25^{\prime} 57,42^{\prime \prime}\end{array}$ & $\begin{array}{l}23.07 .2018 \\
19.08 .2018\end{array}$ \\
\hline
\end{tabular}




\section{Methods}

Animals were observed visually and/or auditively and collected by hand or sampled using a sweep net. Most species were identified in the field, but a male and a female voucher specimen of all species except for the most common ones were preserved in $75 \%$ ethanol. When a species of particular taxonomic interest (e.g. Poecilimon sp. from the elegans group) or unusual morphology (e.g. Pachytrachis gracilis) was concerned, a larger series was collected and preserved in $75 \%$ ethanol for further morphological analyses, and/or in $96 \%$ ethanol for molecular analyses. Material was deposited in the N. Tvrtković and F. Rebrina private collections in Zagreb. Orthoptera were identified using Harz (1969, 1975), Massa et al. (2012), Chobanov \& Heller (2010), Ingrisch \& Pavićević (2010) and Willemse et al. (2018), whereas Mantodea were identified using Harz \& Kaltenbach (1976) and Battiston et al. (2010). Skejo et al. (2018) and Mantodea Species File Online (Отте et al., 2019) were used as references for relevant nomenclature.

\section{RESULTS}

Altogether 45 species of Orthoptera and Mantodea were found in the study area:

Orthoptera

\section{Ensifera}

Tettigoniidae

1. Ephippiger discoidalis Fieber,1853, crvenoglava sedlarka

Material examined: Prapratno, $550 \mathrm{~m}$ a.s.1., 1ڤึ, 24.07.2018, leg. NT; Kuna Konavoska, $670 \mathrm{~m}$ a.s.l., photo Dubravko Dender; Mihanići, $330 \mathrm{~m}$ a.s.1., 1 nymph q, 16.05.2018, basking on the trail table near the tunnel, photo \& leg. NT; near the road Pridvorje - Kuna Konavoska, 570 m, 1 nymph, 06.2006, photo NT;

2. Cyrtaspis scutata (Charpentier,1825), beskrilni staklasti konjic

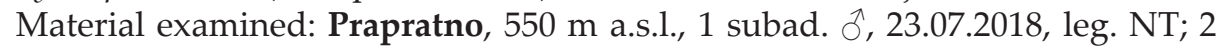
ad. $\widehat{\jmath} \hat{o}+2$ ad. $q$ o, 19.08 .2018 , leg. NT; trail Ljeskovac - fork to Glogovac, 1010 m a.s.1., 20.08.2018, leg. FR; Glogovac, 1000 m a.s.l., 20.08.2018, leg. \& photo FR; Observed specimens: Sv. Luka, 450 m a.s.1., obs. FR;

3. Acrometopa servillea macropoda (Burmeister,1838), dugonogi listokrili konjic Observed specimens: Kuna Konavoska, 700 m a.s.l., obs. FR; Kupljenik, 800 m a.s.1., Ljeskovac, $940 \mathrm{~m}$ a.s.l., obs. FR; Prapratno, $550 \mathrm{~m}$ a.s.1., obs. NT \& FR;

4. Barbitistes ocskayi Charpentier in Ocskay et al., 1850, Ocskayjev ljuskokrili konjic Material examined: Prapratno, 550 m a.s.l., 1q, 23.07.2018, leg. FR;

5. Barbitistes yersini Brunner von Wattenwyl, 1878, Yersinov ljuskokrili konjic

Material examined: trail Ljeskovac - fork to Glogovac, $970 \mathrm{~m}$ a.s.l., $1 \hat{\jmath}$, 21.07.2018, leg. FR; Prapratno, $550 \mathrm{~m}$ a.s.1., 1ㅇ, 23.07.2018, leg. NT;

Observed specimens: Kuna Konavoska, 700 m a.s.1., obs. FR; 
6. Leptophyes intermedia Ingrisch \& Pavićević, 2010, dinarski ljuskokrili konjic

Material examined: Ljeskovac - fork to Glogovac, $1020 \mathrm{~m}$ a.s.1., 10๋, 21.07.2018, leg. FR; Sv. Ilija peak, $1230 \mathrm{~m}$ a.s.1., 1, leg. FR;

7. Leptophyes laticauda (Frivaldszky,1868), riđonogi ljuskokrili konjic

Material examined: Prapratno, $550 \mathrm{~m}$ a.s.1., 1ð̄, 23.07.2018, leg. FR; Ljeskovac fork to Glogovac, $960 \mathrm{~m}$ a.s.1., 23.07.2018, 1ڤ̂, leg. FR; $1010 \mathrm{~m}$ a.s.1., 20.08.2018, 1ㅇ, listening to male calling song from bushes and leg. FR;

8. Poecilimon sp. (undescribed species from elegans group), ljuskokrili konjic

Material examined: Ljeskovac - fork to Glogovac, $1050 \mathrm{~m}$ a.s.1., 1 q, 21.07.2018, leg. FR; fork to Glogovac - Sv. Ilija, $1200 \mathrm{~m}$ a.s.1., 2 ㅇ $q, 21.07 .2018$, leg. FR; Sv. Ilija, $1230 \mathrm{~m}, 1 \delta^{\Uparrow}+1$, 20.08.2018, leg. FR;

9. Poecilimon sp. (from ornatus group), ljuskokrili konjic

Material examined: Prapratno, 550 m a.s.1., 1§̊, 23.07.2018, leg. NT;

10. Tylopsis lilifolia (Fabricius,1793), primorski listokrili konjic

Observed specimens: Pass to Duba Konavoska, 490 m a.s.1., W Stravča, 500 m a.s.l., Prapratno, 550 m a.s.l., Kuna Konavoska, 700 m a.s.l., Kupljenik, 800 m a.s.1., Ljeskovac, 940 m a.s.1., Glogovac, $1000 \mathrm{~m}$ a.s.1., 20.08.2018, all obs. FR \& NT;

11. Saga pedo (Pallas, 1771), konjic vrač

Observed specimens: Sv. Ilija, 1230 m a.s.1., 1q, 20.08.2018, photo FR; in June 2006, 1 nymph photo NT in Kuna Konavoska, 700 m a.s.l. (Buzzetti 2006);

12. Decticus albifrons (Fabricius, 1775), veliki primorski konjic

Observed specimens: Sv Luka, $450 \mathrm{~m}$ a.s.l., Prapratno, $550 \mathrm{~m}$ a.s.1., Kuna Konavoska, $670 \mathrm{~m}$ a.s.1., all obs. FR \& NT;

13. Modestana modesta (Fieber, 1853), skromni livadni konjic

Material examined: Prapratno, $550 \mathrm{~m}$ a.s.l., $1 \hat{\delta}+1+$, 23.07.2018; Ljeskovac, 940 m a.s.1., 2 ㅇ $9,21.07 .2018$; Sv. Ilija peak, $1230 \mathrm{~m}$ a.s.1., 21.07.2018;

Observed specimens: W Stravča, $500 \mathrm{~m}$ a.sl., Sv. Luka, $450 \mathrm{~m}$ a.s.l., Glogovac, $1000 \mathrm{~m}$ a.s.l, all obs. FR \& NT;

14. Pachytrachis frater (Brunner von Wattenwyl, 1882), južni skroviti konjic Material examined: Prapratno, $550 \mathrm{~m}$ a.s.l., 1ठ 23.07.2018, leg. FR; Kuna Konavoska, 670 m a.s.1., 1 , , 21.07.2018, leg. FR; trail Kuna Konavoska - Kupljenik - Ljeskovac, 750 - 850 m a.s.1., $2 \hat{\jmath}+19,21.07 .2018$, leg. FR; trail Ljeskovac - fork to Glogovac, $950 \mathrm{~m}$ a.s.l., $1 \hat{\jmath}$, 21.07.2018, leg. FR;

15. Pachytrachis gracilis (Brunner von Wattenwyl, 1861), sjeverni skroviti konjic Reported as Pachytrachis bosniacus (Buzzetti, 2006: Kuna) 


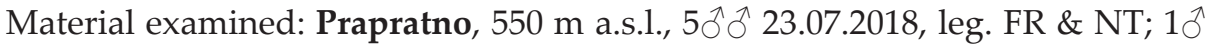

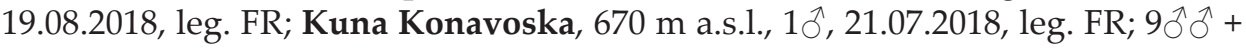
2 우, 21.08.2018, leg. FR \& NT;

16. Pachytrachis striolatus (Fieber,1853), primorski skroviti konjic

Material examined: Ljeskovac, $900 \mathrm{~m}$ a.s.l., 1ðَ, 21.07.2018, leg. FR; Sv. Ilija peak, $1230 \mathrm{~m}$ a.s.1., 2̧ते, 21.07.2018, leg. FR;

Observed specimens: Prapratno, $550 \mathrm{~m}$ a.s.l., nymphs + ad., 19.08.2018, obs. FR; Ljeskovac - Glogovac, 950 - 1040 m a.s.1., nymphs + ad. 21.07.2018, obs. FR; Glogovac - Sv. Ilija, 1040 - 1230 m a.s.1., nymphs + ad., 21.07.2018, obs. FR;

17. Eupholidoptera schmidti (Fieber,1861), crno-zeleni kožokrili konjic

Material examined: Prapratno, $550 \mathrm{~m}$ a.s.1., 23.07.2018, 2 ठ઼, leg. NT; recorded in Kuna Konavoska by Buzzetti;

18. Pholidoptera dalmatica (Krauss, 1879), dinarski kožokrili konjic

Material examined: Mihanići, near the tunnel, $330 \mathrm{~m}$ a.s.l., 1 nymph, 16.05.2018, leg. NT; Prapratno, $550 \mathrm{~m}$ a.s.1., $3 \hat{\jmath} \widehat{\partial}+1+$, 23.07.2018; $2 \hat{\jmath} \widehat{\jmath}, 19.08 .2018$; Ljeskovac, $940 \mathrm{~m}$ a.s.1., 1 , , 21.07.2018; Sv. Ilija peak, $1230 \mathrm{~m}$ a.s.1., $1 \hat{\jmath}+2 q+$, 21.07.2018; all leg. FR \& NT;

19. Pholidoptera fallax (Fischer, 1854), bjelorubi kožokrili konjic

Material examined: Kuna Konavoska, 670 m a.s.l., 1 $1 \delta^{\lambda}$, leg. FR; Prapratno, 550

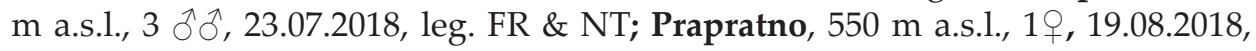
leg. FR;

Observed specimens: Sv. Ilija peak, 1230 m a.s.l., obs. FR;

20. Platycleis albopunctata grisea (Fabricius, 1781), bezgrbi šikarski konjic

Material examined: Glogovac, 1000 m a.s.l., 1 गे, 20.08.2018, leg. FR; Sv. Ilija peak, $1230 \mathrm{~m}$ a.s.1., 1ㅇ, 21.07.2018, leg. FR;

21. Platycleis affinis Fieber, 1853, jednogrbi šikarski konjic

Material examined: Ljeskovac, $940 \mathrm{~m}$ a.s.1., $1 \hat{\delta}+1$, 21.07.2018, leg. FR; Observed specimens: Sv. Luka, $450 \mathrm{~m}$ a.s.l., obs. FR; Prapratno, $550 \mathrm{~m}$ a.s.1., obs. FR;

22. Rhacocleis germanica (Herrich-Schäffer, 1840), konjic grmušar

Material examined: Prapratno, $550 \mathrm{~m}$ a.s.1., $2 \hat{\jmath} \widehat{\jmath}, 23.07 .2018$ and 19.08.2018, leg. FR \& NT; Observed specimens: Kuna Konavoska, 670 m a.s.l., obs. FR \& NT; Sv. Ilija peak, $1230 \mathrm{~m}$ a.s.l., obs. FR \& NT;

23. Sepiana sepium (Yersin, 1854), riđoglavi konjic

Material examined: Prapratno, $550 \mathrm{~m}$ a.s.1., 1 ઈ, 23.07.2018, leg. NT; Observed specimens: Sv. Luka, obs. FR \& NT; Kuna Konavoska, obs. 670 m.a.s.l., obs. FR \& NT; Ljeskovac, obs. FR \& NT; Glogovac, obs. FR \& NT; 
24. Tettigonia viridissima (Linnaeus, 1758), veliki zeleni konjic

Observed specimens: Sv. Luka, 450 m a.s.l., obs. FR \& NT; Prapratno, 550 m a.s.1., obs. NT; Kuna Konavoska, 700 m a.s.1., obs. FR \& NT;

25. Yersinella raymondii (Yersin, 1860), krhka jersinela

Observed specimens: Prapratno, $550 \mathrm{~m}$ a.s.l., obs. FR;

Rhaphidophoridae

26. Dolichopoda araneiformis (Germar in Burmeister,1838), paukoliki spiljski konjic

Material examined: Njivice: Jametina u dolinicah, $470 \mathrm{~m}$ a.s.1., 14.03.2019, a juv. specimen leg. Branko Jalžić;

Observed specimens: Mihanići (tunnel), $330 \mathrm{~m}$ a.s.l., several ad. + subad. specimens 16.05.2018, obs. NT under the bat colony of Myotis myotis/oxygnathus and Rhinolophus ferrumequinum;

*Troglophilus cavicola (Kollar, 1833), obični spiljski konjic

KARAMAN et al., 2011: Špilja Jezero cave, entrance at $880 \mathrm{~m}$ a.s.l. (N slopes of Mt Sniježnica), 1 nymph ô, 30.07.1995, leg. M. Franičević; Glogova jama pit, entrance at $1000 \mathrm{~m}$ a.s.1., 1 nymph , , 28.06.2000, leg. R. Ozimec;

*Troglophilus ovuliformis Karny,1907, primorski spiljski konjic

KARAMAN et al., 2011: Špilja Jezero cave, entrance at $880 \mathrm{~m}$ a.s.l. (N slopes of Mt Sniježnica), 4 nymphs $\widehat{\jmath} \widehat{\jmath}, 16.03 .2002$, leg. R. Ozimec;

Mogoplistidae

27. Arachnocephalus vestitus Costa,1855, ljuskavi šturak grmušar

Observed specimens: Sv. Luka, 450 m a.s.1., 21.08.2018, obs. FR;

Gryllidae

28. Melanogryllus desertus (Pallas, 1771), tamni šturak

Observed specimens: Prapratno, $550 \mathrm{~m}$ a.s.1., listening to male calling song from underneath the Rubus sp. bushes, obs. NT \& FR; identification FR;

29. Gryllomorpha dalmatina (Ocskay, 1833), primorska šturkolika

Observed specimens: Ljeskovac, $940 \mathrm{~m}$ a.s.l., obs. and photo FR;

30. Oecanthus pellucens (Scopoli, 1763), vinogradski prozirni šturak

Observed specimens: W Stravča, $500 \mathrm{~m}$ a.s.1.,18.08.2018, obs. NT \& FR; Sv.

Luka, 450 m a.s.1., 21.08.2018, obs. NT \& FR; Prapratno, 550 m a.s.1., obs. FR \& NT;

\section{Caelifera}

Acrididae

31. Calliptamus italicus (Linnaeus, 1758), talijanski krupnozadi skakavac

Observed specimens: Pass to Duba Konavoska, $490 \mathrm{~m}$ a.s.1., 18.08.2018, W Stravča, 500 m a.s.1., 18.08.2019, Sv. Luka, 450 m a.s.1., 21.08.2018, Prapratno, 550 $\mathrm{m}$ a.sl., Kuna Konavoska, $700 \mathrm{~m}$ a.s.1., all obs. FR \& NT; 
32. Pezotettix giornae (Rossi, 1794), mali smeđi skakavac

Observed specimens: Sv. Luka, 450 m a.sl., 21.08.2018, Prapratno, $550 \mathrm{~m}$ a.s.1., Kuna Konavoska, Ljeskovac, 940 m a.s.l., trail Ljeskovac - fork to Glogovac, 940 - 1040 m a.s.1., Glogovac, 1000 m a.s.l., trail fork to Glogovac - Sv, Ilija, 1040 $1200 \mathrm{~m}$ a.sl., Sv. Ilija peak, $1230 \mathrm{~m}$ a.s.l., all obs. FR \& NT;

33. Anacridium aegyptium (Linnaeus, 1764), egipatska šaška

Observed specimens: Prapratno, 550 m a.s.l., obs. FR \& NT;

34. Arcyptera brevipennis brevipennis (Brunner von Wattenwyl, 1861), dinarski žarki skakavac

Buzzetri (2006) was the first to note this taxon for Mt Sniježnica

Observed specimens: Prapratno, $550 \mathrm{~m}$ a.s.l., trail before Ljeskovac, $930 \mathrm{~m}$ a.s.l., trail Ljeskovac - fork to Glogovac - Sv. Ilija, 1000 - 1150 a.s.1., Sv. Ilija peak, 1230 m a.s.l., all obs. FR \& NT;

*Chorthippus bornhalmi Harz, 1971, primorski livadni skakavac

Observed specimens: trail Kuna Konavoska - Sv. Ilija, 700 - 1230 m a.s.1., July 2005 leg. M. Buzzetti, in field notes as C. brunneus (Buzzetri in litt., 2006);

35. Chorthippus mollis lesinensis (Krauss, 1888), glavati livadni skakavac

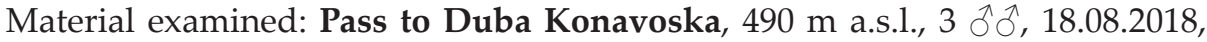
leg. FR; Sv. Luka, 1 ते, 21.08.2018, leg. FR; trail Kupljenik - Ljeskovac, $850 \mathrm{~m}$ a.s.1., $1 \hat{\jmath}, 21.07 .2018$, leg. FR; $1 \hat{\jmath}, 20.08 .2018$, leg. FR; trail Ljeskovac - fork to

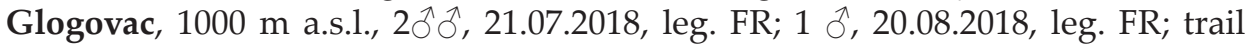
fork to Glogovac - Sv. Ilija, $1100 \mathrm{~m}$ a.s.l., $3 \hat{\partial} \hat{\jmath}, 21.07 .2018$, leg. FR; $2 \hat{\jmath} \hat{\jmath}+2$ 웅, 20.08.2018, leg. FR; Sv. Ilija peak, $1230 \mathrm{~m}$ a.s.1., $2 \hat{\jmath}$, 21.07.2018, leg. FR, $2 \hat{\jmath}$, 20.08.2018, leg. FR;

Observed specimens: Prapratno, $550 \mathrm{~m}$ a.s.l., obs. FR;

36. Omocestus rufipes (Zetterstedt, 1821), crvenozadi travnjački skakavac Material examined: Mihanići (near the tunnel), $330 \mathrm{~m}$ a.s.1., 16.05.2018, leg. NT; Observed specimens: Duba Konavoska, 700 m a.s.l., Sv. Luka, 450 m a.s.l., Glogovac, $1000 \mathrm{~m}$ a.s.1., all obs. FR \& NT;

37. Pseudochorthippus parallelus (Zetterstedt, 1821), obični livadni skakavac

Material examined: Prapratno, $550 \mathrm{~m}$ a.s.1., $1 \hat{\jmath}+1$ 1 , 23.07.2018, leg. NT \& FR; $1 \lesssim+1 q, 14.08 .2018$, leg. NT \& FR; Ljeskovac, $940 \mathrm{~m}$ a.s.1., $3 \hat{\jmath}$, 21.07.2018, leg.

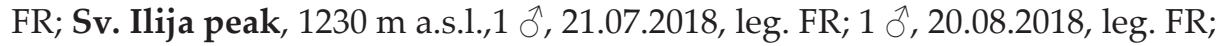

Observed specimens: fork to Glogovac - Sv. Ilija, 1040 - 1200 m a.s.l., obs. FR;

38. Stenobothrus fischeri (Eversmann, 1848), bjeloglavi tamnokrili skakavac

Material examined: Ljeskovac - fork to Glogovac, $1000 \mathrm{~m}$ a.s.1., $3 \widehat{\jmath} \widehat{\jmath}, 21.07 .2018$, leg. FR; Sv. Ilija peak, $1200 \mathrm{~m}$ a.s.1., 1?, 21.07.2018, leg. FR; $1230 \mathrm{~m}$ a.s.1., $1 \hat{\sigma}$, 20.08.2018, leg. FR; 
Observed specimens: Kupljenik, 800 m a.s.1.; Ljeskovac, 940 m a.s.1.; fork to Glogovac - Sv. Ilija, 1100 m a.s.l., all obs. FR;

39. Aiolopus strepens (Latreille, 1804), debelonogi pjegavi skakavac

Observed specimens: Pass to Duba Konavoska, 490 m a.s.l., Sv. Luka, 450 m a.s.1., Duba Konavoska, $458 \mathrm{~m}$ a.s.1., all obs. FR \& NT;

40. Oedipoda caerulescens (Linnaeus,1758), plavokrili kamenjarski skakavac

Observed specimens: Pass to Duba Konavoska, 490 m a.s.1., obs. FR \& NT;

41. Oedipoda meridionalis Ramme, 1913, crvenokrili kamenjarski skakavac

Material examined: Pass to Duba Konavoska, 490 m a.s.1., 18.08.2018, leg. NT \& FR; trail Kuna Konavoska - Kupljenik, 700 m a.s.l.; trail Kupljenik - Ljeskovac, $880 \mathrm{~m}$ a.s.1., leg. FR \& NT;

Observed specimens: Kuna Konavoska 670 m a.s.1., Kupljenik, 800 m a.s.1., Ljeskovac - fork to Glogovac, 1040 m a.s.l., all obs. FR \& NT;

Pamphagidae

42. Prionotropis hystrix (Germar 1817), krški žaboliki skakavac

Buzzetri (2006) was the first to note this taxon for Mt Sniježnica

Observed specimens: trail before Ljeskovac and Ljeskovac, 920 - $940 \mathrm{~m}$ a.s.1., obs. FR \& NT;

Mantodea

Mantidae

1. Ameles heldreichi Brunner von Wattenwyl, 1882, istočna patuljasta bogomoljka Material examined: Pass to Duba Konavoska, $490 \mathrm{~m}$ a.s.1., 18.08.2018, leg. FR; Sv. Ilija, $1230 \mathrm{~m}$ a.s.1., 18.08.2018, leg. FR;

Observed specimens: W Stravča, 500 m a.s.l., Duba Konavoska, 458 m a.s.l., Sv. Luka, $450 \mathrm{~m}$ a.s.l., Prapratno, $550 \mathrm{~m}$ a.s.l., all obs. FR;

2. Mantis religiosa (Linnaeus, 1758), obična bogomoljka

Observed specimens: Pass to Duba Konavoska, 490 m a.s.1., 18.060.2018; Duba Konavoska, $458 \mathrm{~m}$ a.s.1., 18.08.2018; Sv. Luka, $450 \mathrm{~m}$ a.s.1., 21.08.2018, obs. NT \& FR; Prapratno, 550 m, Ljeskovac, 940 m a.s.l., Ljeskovac - fork to Glogovac, 950 - 1050 m a.s.l., fork to Glogovac - Sv. Ilija, 1050 - 1200 m.s.l., Sv. Ilija peak, 1230 m a.s.1., all obs. FR \& NT;

Empusidae

3. Empusa fasciata (Brullé, 1832), krunasta bogomoljka

Observed specimens: Pass to Duba Konavoska, 490 m a.s.1., nymph 18.08.2018, photo FR; near the road Pridvorje - Kuna Konavoska, $570 \mathrm{~m}$ a.s.1., ad. $q$, June 2006, photo NT; NW Mihanići, 330 m a.s.l., obs. Dubravko Dender; 
Of the 45 species observed on the southern slopes of Mt Sniježnica Konavoska, in total, on the foothills below $700 \mathrm{~m}$ a.s.l. we recorded 38 species. The following species occurred only in this, the most thermophilous belt: Ephippiger discoidalis, Barbitistes ocskayi, Decticus albifrons, Eupholidoptera schmidti, Yersinella raymondi, Dolichopoda araneiformis, Arachnocephalus vestitus, Modicogryllus desertus, Oecanthus pellucens, Calliptamus italicus, Anacridium aegyptium, Aiolopus strepens, Oedipoda caerulescens, Empusa fasciata. The findings of two continental species, Pachytrachis gracilis and Pholidoptera fallax, as well as the unexpected finding of Poecilimon sp. from the ornatus group, were restricted to a relatively narrow belt between 500 and $700 \mathrm{~m}$ a.s.l., i.e. Kuna Konavoska plateau with sinkholes and the narrow Prapratno valley before the pass.

On mostly steep, coast-oriented slopes between 700 and $1000 \mathrm{~m}$ a.s.l., we noted 22 species in all. Within this belt there was the uppermost limit of the distribution of several Mediterranean species: Cyrtaspis scutata, Acrometopa servillea macropoda, Barbitistes yersini, Leptophyes laticauda, Tylopsis lilifolia, Pachytrachis frater, Platycleis affinis, Sepiana sepium and Gryllomorpha dalmatina. Heretofore, Prionotropis hystrix was found only in this belt at $950 \mathrm{~m}$ a.s.l. near Ljeskovac, but the mountain trail crosses hot and dry eastern-submediterranean rocky grassland in the early stages of succession, a habitat typical for this species, only at this locality. Accordingly, much wider distribution of this species is expected on Mt Sniježnica.

In the highest belt, just before and around the mountain's peak, Sv. Ilija (1000 $1234 \mathrm{~m}$ a.s.1.), in dry rocky epi-Mediterranean grasslands, we found only 16 species: Poecilimon sp. from elegans group, Leptophyes intermedia, Platycleis albopunctata grisea, Saga pedo, Modestana modesta, Pachytrachis striolatus, Pholidoptera dalmatica, Pholidoptera fallax, Rhachocleis germanica, Arcyptera brevipennis, Stenobothrus fischeri, Pezotettix giornae, Chorthippus mollis lesinensis, Pseudochorthippus parallelus, Ameles heldreichi and Mantis religiosa. The first three species were restricted to this belt.

\section{DISCUSSION}

This study significantly improves the knowledge of the Orthoptera and Mantodea species richness of Mt Sniježnica Konavoska, raising the number of known species from 22 (Buzzetti, 2006 and his field notes, Karaman et al., 2011) to 48 species. Preliminary data on the vertical distribution of Orthoptera and Mantodea on the southern slopes of Mt Sniježnica Konavoska show the expected altitudinal pattern, a decline in species richness with increasing elevation (e.g. KRYŠTUfEK et al., 2008). However, our results also highlight the importance of geomorphological features like isolated peaks (e.g. Sv. Ilija), sinkholes, hollows (e.g. Ljeskovac, Glogovac) and narrow valleys (e.g. Prapratno), for the preservation of rare species. The most important results of the study are the findings of two taxa new to the Croatian fauna.

The first is an undescribed Poecilimon sp. from the elegans group (Fig. 2 a), found in Sv. Ilija peak area, which morphologically corresponds to specimens previously collected in Lovćen pass in Montenegro (HeLler, 1988) and on Mt Orjen (SE Grab) in Bosnia and Herzegovina (Ingrisch \& Pavićević, 2010). A male collected on Mt 
Sniježnica has a subgenital plate rather similar to that of $P$. albolineatus Ingrisch \& Pavićević, 2010 (Fig. 2 a), but, e.g., the white medial line is missing from the tergites and there are about 107 teeth in the stridulatory file.

The second taxon was a larger Poecilimon sp. from the ornatus group (Fig. 2 b), collected in Prapratno valley. It likely belongs to the morphologically very variable $P$. affinis (Frivaldszky, 1868), possibly the subspecies $P$. a. komareki Cejchan, 1953 (Chobanov \& Heller, 2010), the only large Poecilimon known from the neighbouring coastal Mediterranean part of Albania and Montenegro. Both taxa require further research, particularly from the bioacoustic point of view, since their acoustic signals are of the utmost importance for accurate species identification.

On Mt Sniježnica, Leptophyes intermedia (Fig. 2 c) described from Mt Durmitor (Ingrisch \& PavićEvić, 2010) was found in July and August on herbs in dry epiMediterranean grasslands between 1020 and $1230 \mathrm{~m}$ a.s.l. According to PusKás et al. (2018) and SкEjo et al. (2018), this species is endemic to the Dinaric Alps. It was found on the southern slopes of Mt Velebit, as well as on Mosor and Biokovo, at similar altitudes. We observed L. laticauda, a morphologically similar species, up to $960 \mathrm{~m}$ a.s.l., but mainly on bushes or in the crowns of deciduous trees. The findings of L. intermedia and the continental species Platycleis albopunctata grisea and Pholidoptera fallax are the first records of these species in Dubrovnik region.
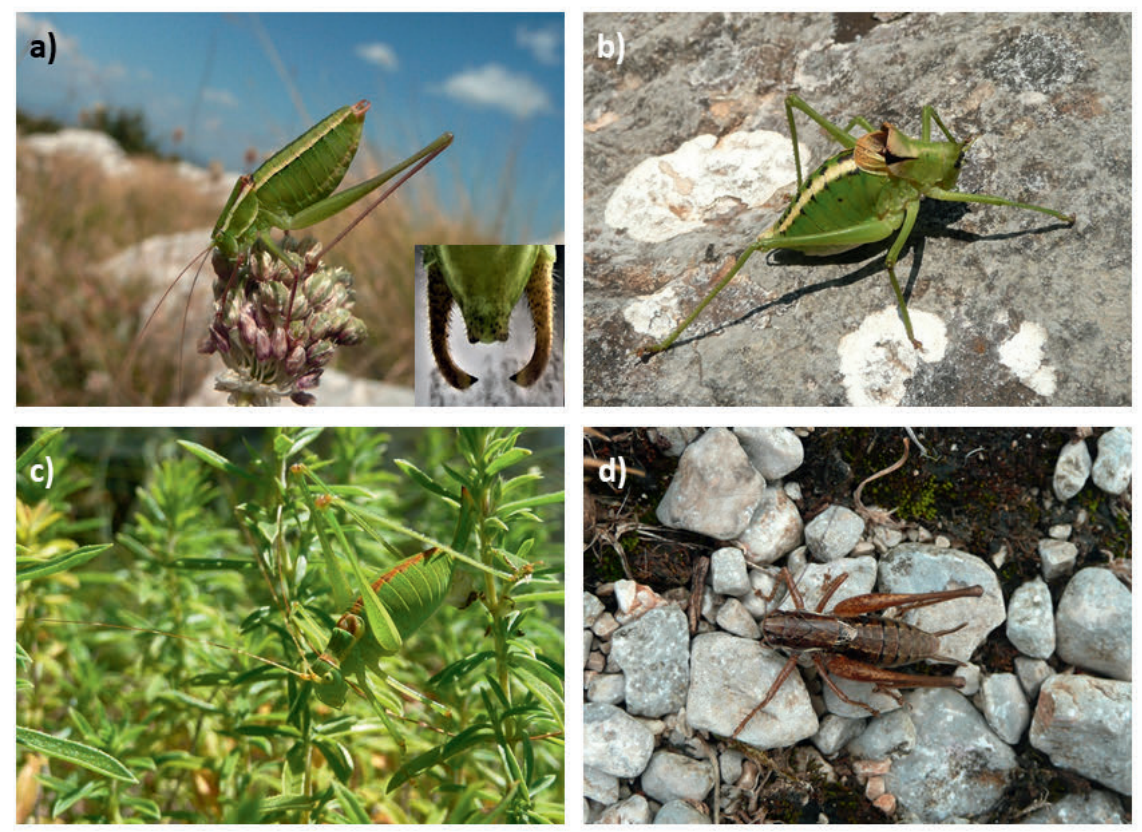

Fig. 2. Notable orthopterans of Mt Sniježnica (Croatia). a) Poecilimon sp. (elegans group) male, with an inserted photograph of the apical part of subgenital plate from below; b) Poecilimon sp. (ornatus group) male; c) Leptophyes intermedia female; d) Pachytrachis gracilis male

(Prapratno near Dubravka population). 
Three sympatrically occurring Pachytrachis species were found in the study area $-P$. frater, $P$. striolatus and $P$. gracilis. The former two species inhabited dry, mostly rocky shrubland and forest edges. On the southern slopes of $\mathrm{Mt}$ Sniježnica, $P$. frater occurred between 600 and $1000 \mathrm{~m}$ a.s.l., whereas $P$. striolatus was found between 900 and $1200 \mathrm{~m}$ a.s.l., sometimes syntopically with $P$. frater. The southern exposure probably allows these thermophilous species (INGRISCH \& Pavićević, 2012) to inhabit relatively high altitudes, P. striolatus reaching the very peak of Mt Sniježnica (Sv. Ilija). The two species also occurred syntopically in the Prapratno valley at $550 \mathrm{~m}$ a.s.l. The topography of this area possibly creates a microclimate similar to that found in the higher portions of the southern slopes, allowing $P$. striolatus to be present here at a lower altitude. On the other hand, $P$. gracilis (Fig. 2 d) was found in more sheltered, somewhat humid forest edges and clearings in the valleys and mountain passes between 500 and $700 \mathrm{~m}$ a.s.l. (i.e. Kuna Konavoska, Prapratno). According to our findings, P. frater had an earlier activity period in the study area than $P$. gracilis and $P$. striolatus; unlike $P$. frater, the latter two species were still found in August, although at this time P. striolatus was already absent from the altitudes below $1100 \mathrm{~m}$ a.s.l.

The shape of the male cerci is a major distinguishing character for $P$. gracilis (Harz, 1969; Ingrisch \& Pavičević, 2010; Ingrisch, 2012; Massa et al., 2012; Willemse et al., 2018). Cercus length in our sample varied between 3.55 and 4.8 $\mathrm{mm}$, according to measurements taken from 15 specimens from two populations (Kuna Konavoska, Prapratno). A peculiar variability of the length, shape and position of male cerci was observed within the populations in the study area. Longer cerci were typically more slender than the shorter ones, with a more prominent attenuation in the middle (Fig. 3 a, b, c). In most identification keys, $P$. gracilis cerci are defined as straight in dorsal view, narrowed in the middle (e.g. Ingrisch \& PAvićEvić, 2010; INGRisch, 2012) and almost parallel (Massa et al., 2012) or slightly diverging (Willemse et al., 2018). In 9 of 15 specimens from the study area, the gap between the apical parts of the diverging cerci was as wide as or wider than cercus length (Fig. 3 a, b). Such cerci were bent laterally in the middle (Fig. 3 a). Nevertheless, titillator shape was typical for the species (HARz, 1969) in all examined specimens, although the length of the apical part varied to some extent (Fig 3. d, e, f), without correlation to the variability of cerci. These findings emphasize the importance of considering the morphological variability of a species when defining its distinguishing characters at a local level.

The unusually high variability of morphological traits in $P$. gracilis population in the study area is probably of interest in uncovering the traces of former microrefugia in different parts of the Dinaric Alps, a part of one of major ice age refugia in the Balkan peninsula (HewitT, 2008). It is possible that the area was a glacial microrefugium, harbouring the original diversity of $P$. gracilis, from which certain morphological variants later colonized other parts of the present distribution. Other potential microrefugia for orthopterans have been identified in the Dinaric Alps (Ivкović et al., 2018). However, this matter can be resolved only with a comprehensive morphological and phylogenetic study, encompassing samples from different parts of the $P$. gracilis distribution. 

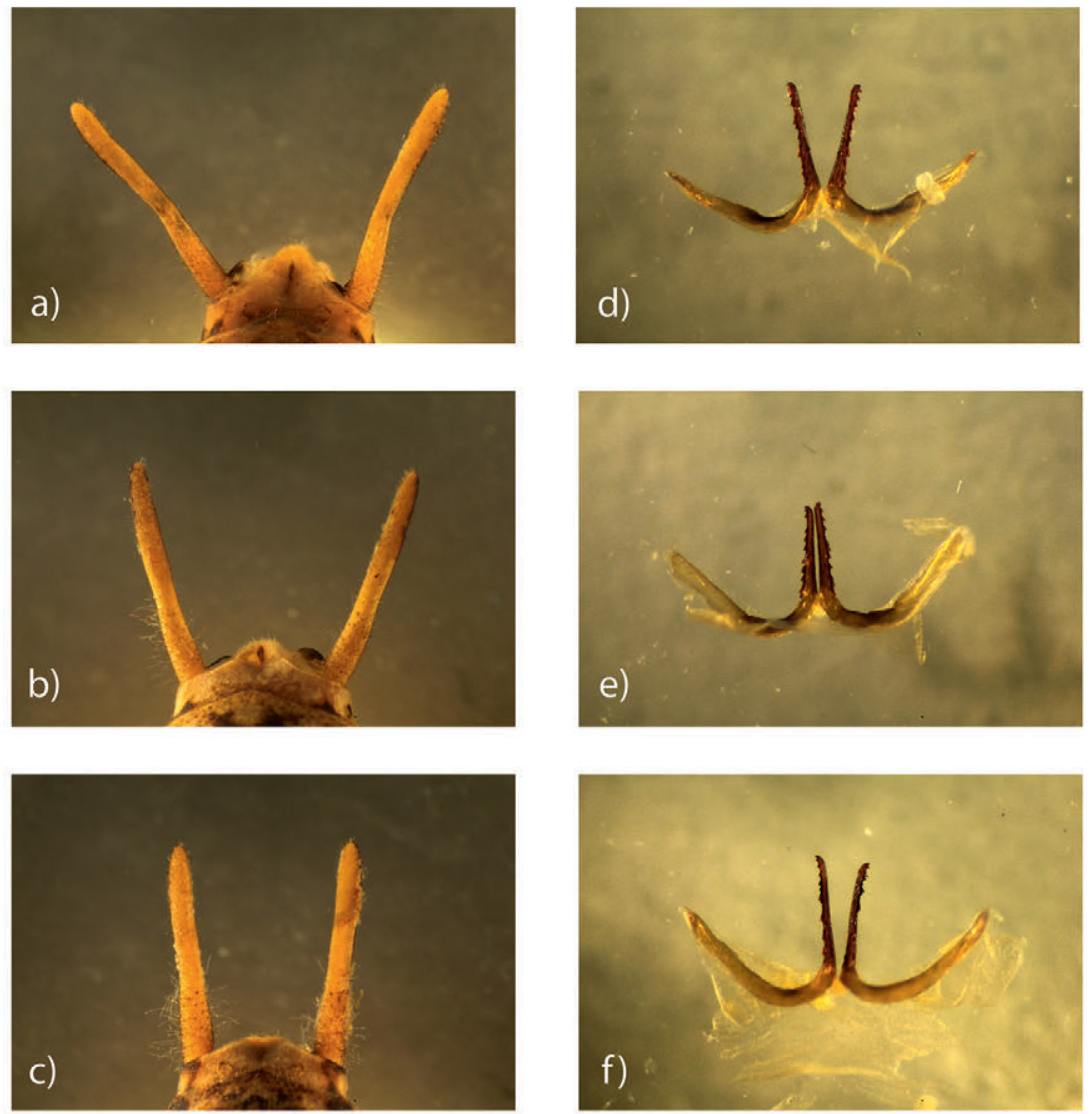

$1 \mathrm{~mm}$

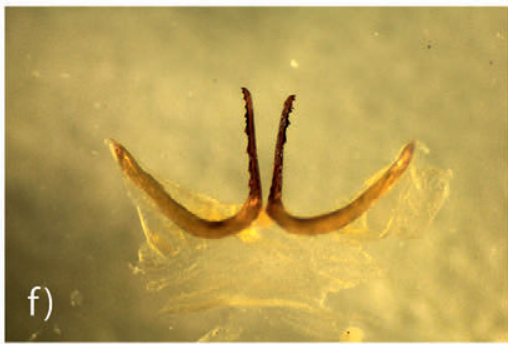

$0,5 \mathrm{~mm}$

Fig. 3. Morphological variability of Pachytrachis gracilis in Kuna Konavoska population (Croatia): an example of three morphological variants (a-d, b-e, c-f), (a-c) male cerci from dorsal view; (d-f) titillators of the same specimens from dorsal view.

Grasshoppers Arcyptera brevipennis and Prionotropis hystrix, found on $\mathrm{Mt}$ Sniježnica, were assessed in the European Red List as Vulnerable (VU) species, while the bush-cricket Pachytrachis frater was listed as Endangered (EN) species, at the European and EU28 level (НоснкіRсн et al., 2016). The bush-cricket Saga pedo is a species included in the Annex of Habitats Directive. Confirmation of the presence of these species on Mt Sniježnica Konavoska is important for the Public Institution for the Protection of Nature and Environment in the Dubrovnik and Neretva County.

\section{ACKNOWLEDGEMENTS}

This study was funded by a research grant awarded by the City of Dubrovnik. We are grateful to Branko Jalžić for his generous help in the field work focusing on cave crickets, to Dr Martina Šašić Kljajo for participating in the field research 
for one day, and to Dubravko Dender for sending us some photographs of local orthopterans and mantids. We are also indebted to the Public Institution for the Protection of Nature and Environment in the Dubrovnik and Neretva County for their support of this research. Finally, we are grateful to the reviewers for improving the quality of this manuscript.

Received May 30, 2019

\section{REFERENCES}

Battiston, R., Picciau, L., Fontana, P. \& Marshall, J., 2010: Mantids of the Euro-Mediterranean area. World Biodiversity Association ONLUS, Verona, $240 \mathrm{pp}$.

Buzzetti, F.M., 2006: Grasshoppers. In: Tvrtкović, N. \& Veen, P. (Eds.), The Dinaric Alps, rare habitats and species. A nature conservation project, Croatia /technical report/. Croatian Natural History Museum \& Royal Dutch, Society for Nature Conservation (KNNV), Zagreb, 1-67.

Chobanov, D. \& Heller, K.-G., 2010: Revision of the Poecilimon ornatus group (Orthoptera: Phaneropteridae) with focus on Bulgaria and Macedonia. European Journal of Entomology, 107, 647-672. doi.org/10.14411/eje.2010.073

Harz, K., 1969: Die Orthopteren Europas I.//The Orthoptera of Europe I. In: Series Entomologica. Vol. 5. Dr. W. Junk, The Hague, 749 pp.

Harz, K., 1975: Die Orthopteren Europas II.//The Orthoptera of Europe II. In: Series Entomologica. Vol. 11. Dr. W. Junk, The Hague, 939 pp.

Harz, K. \& Kaltenbach, A., 1976: Die Orthopteren Europas III.//The Orthoptera of Europe III. In: Series Entomologica. Vol 12. Dr. W. Junk, The Hague, 440 pp.

HeLler, K.-G., 1988: Bioakustik der europäischen Laubheuschrecken. Josef Margraf, Weikersheim, 358 pp.

Hewitt, G. M., 2008: Post-glacial re-colonization of European biota. Biological Journal of the Lennean Society, 68 (1-2), 87-112.

Hochkirch, A., Nieto, A., García Criado, M., Cálix, M., Braud, Y., Buzzetti, F.M., Chobanov, D., Odé, B., Presa Asensio, J.J.,Willemse, L., Zuna-Kratky, T., Barranco Vega, P., Bushell, M.,Clemente, M.E., Correas, J.R., Dusoulier, F., Ferreira, S., Fontana, P., García, M.D., Heller, K-G., Iorgu I.Ș., Ivković, S., Kati, V., Kleukers, R., Krištín, A., Lemonnier-Darcemont, M.,Lemos, P., Massa, B., Monnerat, C., Papapavlou, K.P., Prunier, F., Pushkar, T., Roesti, C.,Rutschmann, F., Şirin, D., Skejo, J., Szövényi, G., Tzirkalli, E., Vedenina, V., Barat Domenech, J., Barros, F., Cordero Tapia, P.J., Defaut, B., Fartmann, T., Gomboc, S., Gutiérrez-Rodríguez, J., Holuša, J., Illich, I., Karjalainen, S., Kočárek, P., Korsunovskaya, O., Liana, A., López, H., Morin, D., Olmo-Vidal, J.M., Puskás, G., Savitsky, V., Stalling, T. \& Tumbrinck, J., 2016: European Red List of Grasshoppers, Crickets and Bush-crickets. Publications Office of the European Union, Luxembourg, $94 \mathrm{pp}$.

INGRISCH, S., 2012: Ilustrated key to the Orthopterous Insects from Durmitor, Montenegro. In: Pavićević, D. \& Perreau, M. (Eds.), Fauna Balkana. Vol. 1. University of Novi Sad, Serbia, 121-149.

Ingrisch, S. \& PAVIĆEvić, D., 2010: Seven new Tettigoniidae (Orthoptera) and a new Blattellidae (Blattodea) from the Durmitor area of Montenegro with notes on previously known taxa. Zootaxa, $2565,1-41$.

Ingrisch, S. \& PavićEvić, D., 2012: Faunistics, distribution and stridulation of orthopteroid insects of the Durmitor plateau and the surrounding canyons. In: Pavićević, D. \& Perreau, M. (Eds.), Fauna Balkana. Vol. 1. University of Novi Sad, Serbia, 13-120.

Ivкоvić, S., PAntović, U. \& SKejo, J., 2018: Ovčar-Kablar Gorge (SW Serbia) - a new hotspot of Orthoptera diversity. Annales de la Société entomologique de France (N.S.), 54(3), 257-272. doi: https://doi.org/10.1080/00379271.2018.1474136

Karaman, I., Hammouti, N., Pavićević, D., Kiefer, A., Horvatović, M. \& Seitz, A., 2011: The genus Troglophilus Krauss, 1879 (Orthoptera: Rhaphidophoridae) in the west Balkans. Zoological Journal of the Linnean Society, 163 (4), 1035-1063. 
Kryštufek, B., JANŽeković, F. \& Režek Bonev, N., 2008: Elevational diversity on two Dinaric mountains. Journal of Natural History, 42 (5-8), 399-408.

MARKović, B., 1971: Osnovna geološka karta SFRJ 1:100 000, list Dubrovnik. Savezni geološki zavod, Beograd + Tumač za list Dubrovnik (K 34-49).

Massa, B., Fontana, P., Buzzetti, F.M., Kleukers, R.M.J.C. \& Odé, B., 2013: Orthoptera. 48. Fauna d'Italia. Calderini, Milano, 563 pp.

Otte, D., Spearman, L. \& Stiewe, M.B.D., 2019: Mantodea Species File Online. Version 5.0/5.0. [29.5.2019]. <http://Mantodea.SpeciesFile.org>.

PusKás, G., NAGY, B. \& SzÖVÉNYI, G., 2018: Faunistical data on the Croatian Orthoptera with four species newly recorded in the country. Annales de la Société entomologique de France (N.S.), 54(6), 539558. doi: 10.1080/00379271.2018.1530071.

Skejo, J., Rebrina, F., SzövÉNY,I G., Puskás, G. \& Tvrtković, N., 2018: The first annotated checklist of Croatian crickets and grasshoppers (Orthoptera: Ensifera, Caelifera). Zootaxa, 4533, 1-95.

Tvrtкović, N., Veen, P. (Eds.), 2006: The Dinaric Alps, rare habitats and species. A nature conservation project, Croatia /technical report/. Croatian Natural History Museum \& Royal Dutch, Society for Nature Conservation (KNNV), Zagreb, 1-67.

Vukelić, J., 2012: Šumska vegetacija Hrvatske (Forest vegetation of Croatia). University of Zagreb, Faculty of Forestry and State Institute for Nature Protection, Zagreb, 403 pp. (In Croatian with English Summary).

Willemse, L.P.M., KLeukers, R.M.J.C. \& OdÉ, B., 2018: The grasshoppers of Greece. EIS Kenniscentrum Insecten \& Naturalis Biodiversity Center, Leiden, 639 pp. 